\title{
Differential Equation-Based Seismic Data Filtering
}

\author{
Jianchao Li and Ken Larner
}

\section{DISCLAIMER}

This report was prepared as an account of work sponsored by an agency of the United States . Neither the United States Government nor any agency thereof, nor any of their Government. Neither the United States Government nor any ags implied, or assumes any legal liability or responsiemployees, makes any warranty, express or implied, or assumes any legal liability or respot, or bility for the accuracy, completeness, or usefulness of any information, apparats, product, process disclosed, or represents that its use would not in or service by trade name, trademark, ence herein to any specific commercial product, process, or service by trade
manufacturer, ur otherwise does not necessarily constitute or imply its endorsement, recommendation, or favoring by the United States Governecessarily state or reflict those of the and opinions of authors expressed herein do

Center for Wave Phenomena

Colorado School of Mines

Golden, Colorado 80401

(303) 273-3557

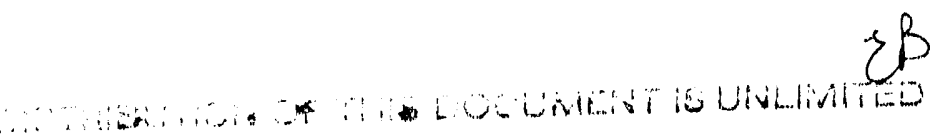




\title{
Differential Equation-Based Seismic Data Filtering
}

\author{
Jianchao Li and Ken Larner
}

\begin{abstract}
Suppressing noise and enhancing useful seismic signal by filtering is one of the important tasks of seismic data processing. Conventional filtering methods are implemented through either the convolution operation or various mathematical transforms. In this paper, we describe a methodology for studying and implementing filters, whicin, unlike those conventional filtering methods, is based on solving differential equations in the time and space domain. We call this kind of filtering differential equation-based filtering (DEBF). DEBF does not require that seismic data be stationary, so filtering parameters can vary with every time and space point. Also, in 2-D and 3-D, DEBF has higher computational efficiency than do conventional multiple-trace filtering methods.

Examples with synthetic and field seismic data show the DEBF methods presented here to be efficient and effective.
\end{abstract}

\section{INTRODUCTION}

Filtering in one form or another is universal in seismic data processing. Geophysicists use it to suppress harmful noise and enhance useful seismic signal, thereby improving the signal-to-noise ratio of seismic data.

Many different 1-D and 2-D filtering methods have been used in seismic data processing, in both the time and the frequency domains. Conventional 2-D moveo't filtering methods for suppressing coherent, dipping events, for example, include timespace domain convolution filtering (Treitel et al., 1967) and frequency-wavenumber $(f-k)$ domain filtering (Wiggins, 1966). Although these methods have been applied successfully in seismic data processing,, some issues remain outstanding.

- Most commonly used filtering methods have directly or indirectly, explicitly or implicitly, a common basic theoretical assumption: the statistical characteristics 
of seismic data do not vary with time and space. Seismic data, however, can be quite non-stationary. The amplitudes of seismic traces gradually attenuate, and the dominant frequency of seismic traces gradually reduces with time; spatial variations of underground media and underground structures must cause spatial variations of the characteristics of seismic data; and within common-midpoint (CMP) gathers, the characteristics of seismic traces also vary in both spatial and temporal directions, among other things because of moveout and of differences in incident angle at reflectors. As a result, the stationarity assumption of typical filtering methods is incorrect.

- To deal with spatial and temporal variations in data, one typically divides the data into several time- and space-windows, within which the characteristics of the filters are kept constant. The final filtered result is a combination of the results obtained from all the time- and space-windows. Thus, the parameters of these filters do not vary arbitrarily with time and space.

The implementation of conventional 2-D time-space domain convolution filtering methods is a process of multitrace convolution. To get well-controlled filtered results, we generally have to use long time and space convolution operators. In such cases, this filtering method generally has lower computational efficiency than does the $f-k$ method. Despite its relative speed, in addition to its problems with handling nonstationary data, the $f-k$ domain filtering has further shortcomings:

- It often produces some harmful artifacts, related to Gibb's phenomena, spatial aliasing, and side effects.

- It often requires a great deal of core computer memory.

- It has some requirements for the length of traces and the number of input traces to avoid wraparound problems and to meet demands of its algorithm.

- It cannot deal well with seismic data whose spatial sampling intervals are variable.

To overcome these shortcomings of conventional filtering methods, new filtering methods, especially in 2-D, have been developed in recent years. These include tau$p$ domain filtering (Noponen and Keeney, 1983), median filtering (Hardage, 1983), depth filtering (McMechan and Sun, 1991), recursive dip filtering (Claerbout, 1985) and adaptive filtering. Tau-p domain filtering can be time-variable, but it is difficult for this method to deal with space variation. While tho two parameters of median filtering-length of running window and number of iterations-can be made to vary with space and time, neither offers fine control for the desired suppression of noise. Depth filtering is based on the idea of wavefield downward and upward continuation to remove near-surface effects, such as the direct wave and ground roll. This method, 
however, is computational ineflicient. Recursive dip filtering method is a form of filtering that can deal with variations of filter parameters with time and space. However, because this method uses a recursive algorithm in the time direction, data must be transposed from trace sequence to time sequence.

The adaptive filtering methods can be divided into two main classes. The first (Anderson and McMechan, 1988) still requires that seismic signal or noise be stationary; the second class (Katz and Katz, 1990), which is suitable for variations of seismic data with both time and space, is based on the joint use of several sets of linear basis filters.

With the increasing emphasis on 3-D seismic exploration, a variety of 3-D seismic data processing techniques, such as $3-\mathrm{D}$ velocity analysis, $3-\mathrm{D}$ dip-moveout (DMO) and 3-D migration, have been developed. As far as we know, however, no truly 3-D filtering methods have been put into practice. Typically, in order to filter 3-D seismic data, one uses existing 2-D filtering methods to filter 2-D sections, first in the inline direction, and then in the crossline direction. When doing so, the 3-D seismic data must be transposed from the inline direction to the crossline direction---a costly step, especially for unstacked data. Other than with these two-pass approaches, many practical difficulties arise in generalizing directly the various above-mentioned methods to 3-D.

In this paper, we shall describe a methodology for studying and developing filtering methods based on differential equations. The key steps of this methodology are: (1) design the filters and set up the filtering equations in the frequency domain or in the $f-k$ domain, (2) transform these equations back into the time or time-space domains, as variable-coefficient differential equations, and (3) use a finite-difference algorithm to solve these equations. The approach we use here is similar to those used in some migration methods. These differential-equation-based filtering (DEBF) methods do not require that seismic data be stationary, so their filtering parameters can arbitrarily vary at every temporal and spatial point. That is, the theoretical foundation of these methods is based on non-stationary processes, and thus better fits typical physical processes.

A.though our main purpose in this paper is to develop 2-D and 3-D DEBF methods, for simplicity we shall start our discussion with the 1-D case.

\section{ONE-DIMENSIONAL PROBLEM}

\subsection{Principle of 1-D DEBF}

Similar to the forms of 1-D Butterworth filters, we define the transfer functions of 1-D filters as follows. 
Low-frequency-pass filters:

$$
H_{1}(x, y, \omega)=\frac{\alpha}{\alpha+\omega^{2 n}}
$$

High-frequency-pass filters:

$$
H_{2}(x, y, \omega)=\frac{\omega^{2 n}}{\alpha+\omega^{2 n}}
$$

and the relation,

$$
H_{1}(x, y, \omega)+H_{2}(x, y, \omega)=1
$$

where $x$ and $y$ are the spatial coordinates; $\omega$ denotes the angular frequency, $\omega=2 \pi f$; $f$ is the frequency; $\alpha$ is a filtering parameter determined by the cut-off frequency; and $n$ is a positive integer that determines the steepness of the boundary between the filter's pass and reject zones. The amplitude curves of $H_{1}(x, y, \omega)$ are shown in Figure 1 , for $n=1,2, \cdots, 10$. From this figure we can see that the larger is $n$, the steeper the boundary between pass and reject zones. Given relation (3), we need discuss only the low-pass filter $H_{1}(x, y, \omega)$. Furthermore, any band-pass filter can be formed by cascading a high- and a low-pass filter. Note, in equation (1), that $H_{1}\left(x, y, \omega_{N}\right)=1 / 2$ gives

$$
\alpha=\omega_{N}^{2 n}=\left(2 \pi f_{N}\right)^{2 n}
$$

where $f_{N}$ is the cut-off frequency. Suppose we use the 1-D low-pass filter defined by equation (1) to filter seismic data. In the frequency-space domain, we have

$$
Q(x, y, \omega)=H_{1}(x, y, \omega) \cdot P(x, y, \omega)
$$

or

$$
\left(\alpha+\omega^{2 n}\right) \cdot Q(x, y, \omega)=\alpha \cdot P(x, y: \omega)
$$

where $P(x, y, \omega)$ is the Fourier transform of the input seismic data $p(x, y, t)$ with respect to $t$, and $Q(x, y, \omega)$ is the filtered output in the frequency-space domain. Although we are discussing 1-D filtering here, the seismic data with which we usually deal are generally 2-D or 3-D. That is why we use as arguments $(x, y, \omega)$. Inverse Fourier transformation of equation (4) into the time-space domain yields 


$$
\left[\alpha(x, y, t)+(-1)^{n} \frac{\partial^{2 n}}{\partial t^{2 n}}\right] \cdot q(x, y, t)=\alpha(x, y, t) \cdot p(x, y, t) .
$$

Here, the filtering parameter $\alpha$ in equation (4) has become the time- and spacedependent coefficient of differential equation (5). Equation (5), with parameter $\alpha(x, y, t)$ varying as desired in space and time, is just the 1-D filtering differential equation we want.

Actually, we can choose a large value of $n$ when solving equation (5). But as we will see, we can benefit conceptually and computationally from choosing $n=1$. Then we obtain

$$
\left[\alpha(x, y, t)-\frac{\partial^{2}}{\partial t^{2}}\right] \cdot q(x, y, t)=\alpha(x, y, t) \cdot p(x, y, t),
$$

with

$$
\alpha(x, y, t)=4 \pi^{2} f_{N}^{2}(x, y, t) .
$$

We see that, through $\alpha(x, y, t)$, the user-specified cut-off frequency $f_{N}$ in general can vary in both time and space. However, for our simplified 1-D argument in this section, we shall use $(t)$ as short-hand for $(x, y, t)$.

\subsection{Algorithm for 1-D DEBF}

Having derived filtering differential equation (5) or (6) to filter seismic data, the key problem lies in finding a stable and efficient method of solving these equations. Mathematically, many algorithms are available to solve this kind of differential equation. Here we use the finite-difference method. Instead of using the ordinary middledifference pattern, we choose (Claerbout, 1985)

$$
\frac{\partial^{2} q(t)}{\partial t^{2}} \approx-\frac{\mathbf{T}}{\Delta t^{2}(\mathbf{I}-\beta \mathbf{T})} \mathbf{q}_{n},
$$

where $\Delta t$ is the time sampling interval, and

$$
\begin{aligned}
\beta & =0.25-1 / \pi^{2}, \\
\mathbf{I} & =[0,1,0], \\
\mathbf{T} & =[-1,2,-1], \\
\mathbf{q}_{n} & =\left[q_{n-1}, q_{n}, q_{n+1}\right]^{T}, \\
q_{n} & =q(n \Delta t), \quad n=1,2,3, \cdots, L,
\end{aligned}
$$


and where $L$ denotes the maximum length, in sample points, of seismic traces to be processed. Substituting equation (8) and (9) into equation (6), we find

$$
\begin{gathered}
{\left[\alpha_{n} \mathbf{I}+\frac{\mathbf{T}}{\Delta t^{2}(\mathbf{I}-\beta \mathbf{T})}\right] \cdot \mathbf{q}_{n}=\alpha_{n} \mathbf{I} \cdot \mathbf{p}_{n}} \\
n=1,2,3, \cdots, L
\end{gathered}
$$

where

$$
\begin{aligned}
\alpha_{n} & =\alpha(n \Delta t), \\
p_{n} & =\left[p_{n-1}, p_{n}, p_{n+1}\right]^{T}, \\
p_{n} & =p(n \Delta t), \\
p_{0} & =0, \quad p_{L+1}=0 .
\end{aligned}
$$

If let

$$
\begin{aligned}
\theta_{n} & =\Delta t^{2} \alpha_{n}=4 \pi^{2} \Delta t^{2} f_{N n}^{2}, \\
f_{N n} & =f_{N}(n \Delta t),
\end{aligned}
$$

equation (10) can be written as

$$
\begin{aligned}
{\left[\theta_{n} \mathbf{I}+\left(1-\theta_{n} \beta\right) \mathbf{T}\right] \cdot \mathbf{q}_{n} } & =\theta_{n}(\mathbf{I}-\beta \mathbf{T}) \cdot \mathbf{p}_{n} \\
n & =1,2,3, \cdots, L .
\end{aligned}
$$

We may express this equation in matrix terms as

$$
\mathbf{A} \cdot \mathbf{q}=\mathbf{B}
$$

where

$$
\mathrm{B}=\mathrm{C} \cdot \mathrm{p}
$$




$$
\begin{aligned}
& \mathbf{A}=\left[\begin{array}{ccccccc}
b_{1} & c_{1} & 0 & 0 & \cdots & 0 & 0 \\
a_{2} & b_{2} & c_{2} & 0 & \cdots & 0 & 0 \\
0 & a_{3} & b_{3} & c_{3} & \cdots & 0 & 0 \\
\vdots & \vdots & \vdots & \vdots & \ddots & \vdots & \vdots \\
0 & 0 & 0 & 0 & \cdots & a_{L} & b_{L}
\end{array}\right] \\
& \mathbf{C}=\left[\begin{array}{ccccccc}
e_{1} & f_{1} & 0 & 0 & \cdots & 0 & 0 \\
d_{2} & e_{2} & f_{2} & 0 & \cdots & 0 & 0 \\
0 & d_{3} & e_{3} & f_{3} & \cdots & 0 & 0 \\
\vdots & \vdots & \vdots & \vdots & \ddots & \vdots & \vdots \\
0 & 0 & 0 & 0 & \cdots & d_{L} & e_{L}
\end{array}\right] \\
& \mathbf{q}=\left[q_{1}, q_{2}, q_{3}, \cdots, q_{L}\right]^{T}, \\
& \mathbf{p}=\left[p_{1}, p_{2}, p_{3}, \cdots, p_{L}\right]^{T}, \\
& a_{n}=c_{n}=\theta_{n} \beta-1 \text {, } \\
& b_{n}=\theta_{n}-2\left(\theta_{n} \beta-1\right) \text {, } \\
& d_{n}=f_{n}=\theta_{n} \beta, \\
& e_{n}=\theta_{n}(1-2 \beta) \text {. }
\end{aligned}
$$

Equation (12) is a differential equation-based, implicit finite-difference implementation of 1-D filtering. It can be seen that equation (12) is a diagonally dominant tridiagonal system of equations, so, we can use a well-known fast algorithm to solve this special system (Clearbout, 1985). This algorithm ensures a solution of equation (12) that is stable and efficient. The tridiagonal nature of the equation is a result of the choice $n=1$. To get sharper cut-off action, we see in Figure 1 that we would have to choose the larger value of $n$ in equation (5). If we did so, however, the resulting system of equations would no longer be tridiagonal, but rather a system of equations with progressively increasing number of nonzero diagonals as $n$ increases. As a result, we would lose the computational advantage of the tridiagonal system. Fortunately, however, we would have some other efficient solutions, such as the Cholesky decomposition method. The computational cost of this solution grows only linearly with the size $L$. By solving equation (12) with the user-specified cut-off frequency $f_{N}(x, y, t)$, differential equation-based 1-D filtering of seismic data can be implemented trace-by-trace. 


\subsection{Examples with synthetic dara}

To test the effectiveness of the 1-D filtering method presented in this section, we shall study a synthetic data example. The wavelet we used to create synthetic seismic sections is defined by

$$
s(t)=\frac{20}{t} e^{-2000 t^{2}} \sin (30 \pi t) \cos (62.5 \pi t) .
$$

Frequencies of this wavelet are in the range of typical seismic signals.

Figure $2 \mathrm{a}$ is a synthetic seismic section consisting of two horizontal reflections with low-frequency $(1-10 \mathrm{~Hz})$ "noise" superimposed. Figure $2 \mathrm{~b}$ shows the amplitude spectra of four traces from Figure $2 \mathrm{a}$. The results of applying time- and space-variable filtering of the data in Figure 2a are shown in Figure 3. The low cut-off frequency in this test is governed by the following parameters,

at the second trace: $t 200 f 1, t 400 f 10$ and $t 450 f 40$,

and at the 19th trace: $t 200 f 40, t 400 f 10$ and $t 450 f 1$.

Here, for example, $t 200 f 1, t 400 f 10$ and $t 450 f 40$ means that before $200 \mathrm{~ms}$, the low cut-off frequency is $1 \mathrm{~Hz}$; at $400 \mathrm{~ms}, 10 \mathrm{~Hz}$; and after $450 \mathrm{~ms}, 40 \mathrm{~Hz}$. The low cut-off frequencies at all other points are obtained by linear interpolation. Laterally, these cut-off frequencies are also interpolated linearly between traces along which values are specified. Figure $3 \mathrm{a}$ is the filtered section, and Figure $3 \mathrm{~b}$ is the so-called noise section, the difference between Figure $2 \mathrm{a}$ and Figure 3a. Figure $3 \mathrm{c}$ shows the amplitude spectra for the 1st, 5th, 10th and 15th traces of Figure 3a. The results in these three figures are just what we expect.

\section{TWO-DIMENSIONAL PROBLEM}

\subsection{Principle of 2-D DEBF}

Having introduced the conception of DEBF with the 1-D case, we now discuss 2-D DEBF. In the $f-k$ domain, we define the transfer functions of $2-D$ moveout filters we use as follows.

High-dip-pass filter:

$$
H_{1}\left(k_{x}, y, \omega\right)=\frac{\alpha}{\alpha+\omega^{2} / i k_{x}}
$$

Low-dip-pass filter:

$$
H_{2}\left(k_{x}, y, \omega\right)=\frac{\omega^{2} / i k_{x}}{\alpha+\omega^{2} / i k_{x}}
$$


and the relation,

$$
H_{1}\left(k_{x}, y, \omega\right)+H_{2}\left(k_{x}, y, \omega\right)=1
$$

where $k_{x}$ is the wavenumber in the $x$-direction, and $\alpha$ is a 2-D filtering parameter determined by the user-specified cut-off "dip" or by a combination of the user-specified dominant frequency and cut-off apparent velocity.

Actually, we might have defined other forms for the transfer function, such as $H_{1}\left(k_{x}, y, \omega\right)=\alpha /\left(\alpha+\omega^{2} / k_{x}^{2}\right)$. While such a choice would give a response that is strictly a function of slope $k_{x} / \omega$, we choose the above forms for simplicity of development, computational efficiency, and stability. Given relation (15), we need only discuss the high-dip-pass filter $H_{1}\left(k_{x}, y, \omega\right)$. Its amplitude and phase spectra are, respectively,

$$
\left|H_{1}\left(k_{x}, y, \omega\right)\right|=\frac{1}{\sqrt{1+\omega^{4} / \alpha^{2} k_{x}^{2}}}
$$

and

$$
\theta_{1}\left(k_{x}, y, \omega\right)=\tan ^{-1}\left(\frac{\omega^{2}}{\alpha k_{x}}\right) .
$$

Letting $\left|H_{1}\left(k_{x}, y, \omega\right)\right|=\varepsilon$, some chosen amplitude level, then from equation (16)

$$
k_{x}= \pm \frac{\varepsilon}{\sqrt{1-\varepsilon^{2}}} \cdot \frac{\omega^{2}}{\alpha}
$$

where $\varepsilon$ is a positive constant, less than 1. For different values of $\varepsilon$, equation (18) describes different parabolas that are symmetric with respect to both the $\omega$ - and $k_{x^{-}}$ axis and pass through the origin. That is, the contours of amplitude spectrum of the 2-D filter $H_{1}\left(k_{x}, y, \omega\right)$ defined by equation (13) are symmetric parabolas. If, further, we let $\varepsilon=1 / \sqrt{2}$, we get a special contour,

$$
k_{x}= \pm \frac{\omega^{2}}{\alpha}
$$

This is the expression of the half-power contour of $H_{1}\left(k_{x}, y, \omega\right)$. Now let us see the relationship between $H_{1}\left(k_{x}, y, \omega\right)$ and the response of an ideal dip filter. In. Figure $4 \mathrm{a}$, covering only the range $\omega \geq 0$ and $k_{x} \geq 0$, the straight line denotes the boundary $k_{x}=\omega / V_{N}$ between the pass and reject zone of an ideal dip filter, while the parabola is the boundary of the filter $H_{1}\left(k_{x}, y, \omega\right)$ defined by equation (19). Here, $V_{N}$ is the cutoff apparent velocity. Therefore, when using the filter $H_{1}\left(k_{x}, y, \omega\right)$ as a dip filter, we 
are, in effect, replacing the straight-line boundary of the ideal filter with the parabolic boundary. To get desired filtered results, we should make the parabolic boundary of $H_{1}\left(k_{x}, y, \omega\right)$ as close to the straight line boundary of the ideal dip filter as possible within the range of $\omega$ and $k_{x}$ of interest. This can be done by minimizing the area between the straight line and the parabolic boundary. Over the frequency range of interest, for example, if the range is from 0 to $f_{\max }$, we get an expression for the filtering parameter $\alpha$,

$$
\alpha=2^{4 / 3} \pi f_{N} V_{N} .
$$

Here, we take $f_{N}=f_{\max } / 2$ as the user-specified dominant frequency. If we use the concept of apparent velocity to characterize 2-D filters, $H_{1}\left(k_{x}, y, \omega\right)$ is a lowapparent-velocity-pass filter. Figure $4 \mathrm{~b}$ shows three contours, $\varepsilon=0.707,0.6$ and 0.5 , of the amplitude spectrum $\left|H_{1}\left(k_{x}, y, \omega\right)\right|$, when $\alpha$ takes the form of equation (20), for $f_{N}=35 \mathrm{~Hz}$ and $V_{N}=3000 \mathrm{~m} / \mathrm{s}$. We see that $H_{1}\left(k_{x}, y, \omega\right)$ is an approximation of the ideal dip filter, and the transition of its boundary from the pass to the reject zone is smooth.

Using $H_{1}\left(k_{x}, y, \omega\right)$ defined by equation (13) and (20) to filter seismic data, the filtering equation in the $f-k$ domain can be expressed as

$$
Q\left(k_{x}, y, \omega\right)=H_{1}\left(k_{x}, y, \omega\right) \cdot P\left(k_{x}, y, \omega\right)
$$

or

$$
\left(i \alpha k_{x}+\omega^{2}\right) Q\left(k_{x}, y, \omega\right)=i \alpha k_{x} P\left(k_{x}, y, \omega\right),
$$

where $P\left(k_{x}, y, \omega\right)$ is the Fourier transformation of the input seismic data $p(x, y, t)$ with respect to $x$ and $t ; Q\left(k_{x}, y, \omega\right)$ is the filtered result in the $f_{-k}$ domain. Inverse Fourier transformation of equation (21) into the time-space domain yields

$$
\left[\alpha(x, y, t) \frac{\partial}{\partial x}-\frac{\partial^{2}}{\partial t^{2}}\right] q(x, y, t)=\alpha(x, y, t) \frac{\partial p(x, y, t)}{\partial x} .
$$

If the seismic data with which we deal are only 2-D, we can use the short-hand $(x, t)$ instead of $(x, y, t)$ for the arguments. Thus, the above equation becomes

$$
\left[\alpha(x, t) \frac{\partial}{\partial x}-\frac{\partial^{2}}{\partial t^{2}}\right] q(x, t)=\alpha(x, t) \frac{\partial p(x, t)}{\partial x}
$$

with

$$
\alpha(x, t)=2^{4 / 3} \pi f_{N} V_{N}(x, t) .
$$

Here, $q(x, t)$ is the filtered output in the time-space domain, and the filtering parameter $\alpha(x, t)$, determined by $f_{N}$ and $V_{N}(x, t)$, is a function of both time and space, i.e. it can vary arbitrarily in both temporal and spatial directions. Equation (22) is just the variable-coefficient differential equation we want for 2-D high-dip-pass, or 
low-apparent-velocity-pass, filtering. To solve this partial differential equation, we use the following conditions:

$$
\begin{aligned}
& \left.p(x, t)\right|_{x=-\Delta x}=\left.\eta \cdot p(x, t)\right|_{x=0}, \\
& \left.q(x, t)\right|_{x=-\Delta x}=\left.\eta \cdot q(x, t)\right|_{x=0}, \\
& \left.q(x, t)\right|_{t=0}=0,\left.\quad q(x, t)\right|_{t=t_{\text {max }}}=0,
\end{aligned}
$$

where $\eta$ is a constant, $0 \leq \eta \leq 1, \Delta x$ is the trace spacing, and $x=0$ is the location of the first trace in the seismic data.

\subsection{Algorithm for 2-D DEBF}

Using the modified Crank-Nicholson difference pattern (Claerbout, 1985) to solve the determined problem (22) and (24), we obtain the following difference equation

$$
\begin{gathered}
\frac{\alpha_{m}(n)}{\Delta x} \mathbf{I}\left[\mathbf{q}_{m}(n)-\mathbf{q}_{m-1}(n)\right]+\frac{\mathbf{T}}{2 \Delta t^{2}(\mathbf{I}-\beta \mathbf{T})}\left[\mathbf{q}_{m}(n)+\mathbf{q}_{m-1}(n)\right] \\
=\frac{\alpha_{m}(n)}{\Delta x} \mathbf{I}\left[\mathbf{p}_{m}(n)-\mathbf{p}_{m-1}(n)\right] \\
n=1,2,3, \cdots, L, \quad m=0,1,2, \cdots, M
\end{gathered}
$$

and the discrete expressions of the conditions for determining solution

$$
\begin{aligned}
\mathbf{p}_{-1} & =\eta \cdot \mathbf{p}_{0}, \\
\mathbf{q}_{-1} & =\eta \cdot \mathbf{q}_{0}, \\
q_{m}(0) & =0, \quad q_{m}(L+1)=0,
\end{aligned}
$$

where $\Delta t$ is the time sampling interval, $L$ is the maximum length of seismic traces in sample points, $M$ is the maximum number of traces processed, and

$$
\begin{aligned}
\mathbf{I} & =[0,1,0] \\
\mathbf{T} & =[-1,2,-1] \\
\alpha_{m}(n) & =\alpha(m \Delta x, n \Delta t), \\
\beta & =0.25-1 / \pi^{2} \\
q_{m}(n) & =q(m \Delta x, n \Delta t) \\
p_{m}(n) & =p(m \Delta x, n \Delta t), \\
\mathbf{q}_{m}(n) & =\left[q_{m}(n-1), q_{m}(n), q_{m}(n+1)\right]^{T}
\end{aligned}
$$




$$
\begin{aligned}
\mathbf{p}_{m}(n) & =\left[p_{m}(n-1), p_{m}(n), p_{m}(n+1)\right]^{T} \\
\mathbf{q}_{m} & =\left[q_{m}(1), q_{m}(2), q_{m}(3), \ldots, q_{m}(L)\right]^{T} \\
\mathbf{p}_{m} & =\left[p_{m}(1), p_{m}(2), p_{m}(3), \ldots, p_{m}(L)\right]^{T}
\end{aligned}
$$

Let

$$
\theta_{m}(n)=2 \Delta t^{2} \alpha_{m}(n) / \Delta x=2^{7 / 3} \pi \Delta t^{2} f_{N} V_{N m}(n)
$$

Then the difference equation (25) becomes

$$
\begin{gathered}
{\left[\theta_{m}(n) \mathbf{I}+\left(1-\theta_{m}(n) \beta\right) \mathbf{T}\right] \cdot q_{m}(n)=\left[\theta_{m}(n) \mathbf{I}-\left(1+\theta_{m}(n) \beta\right) \mathbf{T}\right] \cdot q_{m-1}(n)} \\
+\theta_{m}(n)(\mathbf{I}-\beta \mathbf{T})\left[p_{m}(n) \cdots p_{m-1}(n)\right], \\
n=1,2,3, \cdots, L, \quad m=0,1,2, \cdots, M .
\end{gathered}
$$

If we let

$$
\begin{aligned}
& \mathbf{A}=\left[\begin{array}{ccccccc}
b_{1} & a_{1} & 0 & 0 & \cdots & 0 & 0 \\
a_{2} & b_{2} & a_{2} & 0 & \cdots & 0 & 0 \\
0 & a_{3} & b_{3} & a_{3} & \cdots & 0 & 0 \\
\vdots & \vdots & \vdots & \vdots & \ddots & \vdots & \vdots \\
0 & 0 & 0 & 0 & \cdots & a_{L} & b_{L}
\end{array}\right], \\
& \mathbf{B}=\left[\begin{array}{ccccccc}
d_{1} & c_{1} & 0 & 0 & \cdots & 0 & 0 \\
c_{2} & d_{2} & c_{2} & 0 & \cdots & 0 & 0 \\
0 & c_{3} & d_{3} & c_{3} & \cdots & 0 & 0 \\
\vdots & \vdots & \vdots & \vdots & \ddots & \vdots & \vdots \\
0 & 0 & 0 & 0 & \cdots & c_{L} & d_{L}
\end{array}\right] \\
& \mathbf{C}=\left[\begin{array}{ccccccc}
f_{1} & e_{1} & 0 & 0 & \cdots & 0 & 0 \\
\epsilon_{2} & f_{2} & e_{2} & 0 & \cdots & 0 & 0 \\
0 & e_{3} & f_{3} & e_{3} & \cdots & 0 & 0 \\
\vdots & \vdots & \vdots & \vdots & \ddots & \vdots & \vdots \\
0 & 0 & 0 & 0 & \cdots & e_{L} & f_{L}
\end{array}\right] \\
& a_{n}=\theta_{m}(n) \beta-1 \text {, } \\
& b_{n}=\theta_{m}(n)+2\left(1-\theta_{m}(n) \beta\right) \text {, }
\end{aligned}
$$




$$
\begin{aligned}
& c_{n}=1+\theta_{m}(n) \beta, \\
& d_{n}=\theta_{m}(n)-2\left(1+\theta_{m}(n) \beta\right), \\
& e_{n}=\theta_{m}(n) \beta, \\
& f_{n}: \theta_{m}(n)(1-2 \beta),
\end{aligned}
$$

equation (28) can be expressed in matrix terms as

$$
\begin{gathered}
\mathbf{A q}_{m}=\mathbf{B} \mathbf{q}_{m-1}+\mathbf{C}\left(\mathbf{p}_{m}-\mathbf{p}_{m-1}\right) \\
m=0,1,2, \cdots, M
\end{gathered}
$$

This equation is an example of differential equation-based implementation of 2-D filtering. As for the 1-D example, equation (29) is a diagonally dominant tridiagonal system of equations, so it too can be solved by using a fast algorithm. Also, from equation (28) and (29), to get the filtered result at a given point, only six adjacrnt points on two adjacent traces are involved; thus to obtain one output trace, only two input traces are used. So, on the one hand, computational operators of this kind of filter are quite short in both temporal and spatial directions and computational efficiency is high; on the other hand, through use of the implicit approach in the time direction and the explicitly recursive approach in the space direction, we, in effect, achieve an extended 2-D impulse response.

So far, we have been discussing 2-D high-dip-pass filtering. By relation (15), instead of applying directly $H_{2}\left(k_{x}, y, \omega\right)$ defined by equation $(14)$, we subtract the filtered result of 2-D high-dip-pass filtering from the original input seismic data to get the result of $2-D$ low-dip-pass filtering.

\subsection{Examples with synthetic and field data}

We demonstrate the action of the 2-D filtering method first on synthetic and then on field seismic data. Figure 5a contains horizontal events and events with moveout we shall call small, moderate, and large. Figure $5 \mathrm{~b}$ shows a time- and spacevariable high-apparent-velocity-pass (or low-dip-pass) filtered result of Figure 5a. In this section any given dipping event has been passed at some time and space points and rejected at the nthers. In this exampie, the dominant frequency is $20 \mathrm{~Hz}$, and the cut-off apparent-velocity $V_{N}(x, i)$ was governed by the following parameters,

at the first trace: $t 1500 V 5500$ and $t 3510 V 50$,

at the 250th trace: $1.500 \mathrm{~V} 5500$ and $t 2510 \mathrm{~V} 50$,

and at 500 th trace: $t 1500 V 5500$ and $t 1510 \mathrm{~V} 50$.

Here, for example, $t 1500 \mathrm{~V} 5500$ and $t 3510 \mathrm{~V} 50$ means that before $1510 \mathrm{~ms}$ : the cut-off apparent-velocity is $5500 \mathrm{~m} / \mathrm{s}$ and after $3510 \mathrm{~ms}, 50 \mathrm{~m} / \mathrm{s}$. The values of $V_{N}$ ai all other points are obtained by linearly interpolation. 
Figure $5 \mathrm{c}$ is a detail, corresponding to the box position in Figure 5a, of the highapparent-velocity-pass fil' ared results of Figure 5a obtained by using 2-D DEBF, and Figure $5 \mathrm{~d}$ is that obtained by $f-k$ filtering. Our purpose is to reject the steep events and to pass the horizontal events and those three events witu small dips. The cut-off boundary just coincides with the events with moderate dip. Comparing Figure $5 \mathrm{c}$ with $5 \mathrm{~d}$, we see that $f-k$ filter has a sharper cut-off boundary than does DEBF, but has some artifacts on the side, while DEBF does not.

The second example is a demonstration of noise suppression on a field shot record. Figure 6a shows the raw data, which are contaminated by large-moveout coherent noise in the upper part, and Figure $6 \mathrm{~b}$ shows the 2-D time- and space-variable DEBF filtered result of Figure 6a showing suppression of the coherent noise. Here, $f_{N}=$ $30 \mathrm{~Hz}$ and $V_{N}(x, t)$ was governed by the following parameters,

at the first trace: $t 700 \mathrm{~V} 2700$ and $t 1000 \mathrm{~V} 3500$,

at the 32 nd trace: $t 1500 V 3500$,

at the 52nd trace: ${ }^{+800 V 2200}$ and $1500 \mathrm{~V} 3500$,

and at the $i$ nd trace: $t 1100 \mathrm{~V} 1700$ an $t 2000 \mathrm{~V} 3500$.

The final 2-D filter example is a common-offset field seismic section. The raw section (Figure 7a), especially the right part of this section, has much steeply dipping background noise. In Figure $7 \mathrm{~b}$, this noise has been reduced, and the signal-to-noise ratio thereby has been improved.

Clearly, although we do not show it here, this kind of filtering method could also be used to separrte down-going and up-going waves in VSP data.

For a test data set consisted of 1.024 traces, each containing 1024 samples, we have compared the computational efficiency of the 2-D DEBF program written in FORTRAN with that of an $f-k$ filtering program in $C$ on an IBM RS/6000 workstation. Under the same conditions, the 2-D DEBF method took 1 minute and 2l) seconds, winile the $f$ - $k$-domain filtering method in the SU system took 1 minute and 37 seconds. This test suggests that speeds of the 2-D DEBF and $f-k$ methods are comparable.

\section{THREE-DIMENSIONAL PROBLEM}

\subsection{Principle and algorithm of 3-D DEBF}

In the previous section, we defined the ingh-dip-pass filter and discussed its implementation in detail. One way for us to define the transfer function of a 3-D high-dip-pass filter is as follows,

$$
H\left(k_{x}, k_{y}, \omega\right)=\frac{\alpha_{x}}{\alpha_{x}+\omega^{2} / i k_{x}} \cdot \frac{\alpha_{y}}{\alpha_{y}+\omega^{2} / i k_{y}}=H_{1}\left(k_{x}, \omega\right) \cdot H_{1}\left(k_{y}, \omega\right),
$$


where both $H_{1}\left(k_{x}, \omega\right)$ and $H_{1}\left(k_{y}, \omega\right)$ are 2-D high-dip-pass filters, where $H_{1}\left(k_{x}, \omega\right)$ is used in the x-direction (say, the inline direction), while $H_{1}\left(k_{y}, \omega\right)$ is used in the ydirection (say, the crossline direction). Using $H\left(k_{x}, k_{y}, \omega\right)$ to filter 3-D seismic data, we obtain the filtering equation in the $f-k$ domain

$$
\begin{array}{r}
Q\left(k_{x}, k_{y}, \omega\right)=H\left(k_{x}, k_{y}, \omega\right) \cdot P\left(k_{x}, k_{y}, \omega\right) \\
\quad=H_{1}\left(k_{y}, \omega\right) \cdot H_{1}\left(k_{x}, \omega\right) \cdot P\left(k_{x}, k_{y}, \omega\right) .
\end{array}
$$

If we let

$$
G\left(k_{x}, k_{y}, \omega\right)=H_{1}\left(k_{x}, \omega\right) \cdot P\left(k_{x}, k_{y}, \omega\right)=\frac{\alpha_{x}}{\alpha_{x}+\omega^{2} / i k_{x}} \cdot P\left(k_{x}, k_{y}, \omega\right),
$$

then equation (31) becomes

$$
Q\left(k_{x}, k_{y}, \omega\right)=H_{1}\left(k_{y}, \omega\right) \cdot G\left(k_{x}, k_{y}, \omega\right)=\frac{\alpha_{y}}{\alpha_{y}+\omega^{2} / i k_{y}} \cdot G\left(k_{x}, k_{y}, \omega\right) .
$$

Inverse Fourier transformation of equation (32) and (33) into the time-space domain yields a system of 3-D high-dip-pass filtering differential equations,

$$
\left[\alpha_{x}(x, y, t) \frac{\partial}{\partial x}-\frac{\partial^{2}}{\partial t^{2}}\right] g(x, y, t)=\alpha_{x}(x, y, t) \frac{\partial p(x, y, t)}{\partial x}
$$

and

$$
\left[\alpha_{y}(x, y, t) \frac{\partial}{\partial y}-\frac{\partial^{2}}{\partial t^{2}}\right] q(x, y, t)=\alpha_{y}(x, y, t) \frac{\partial g(x, y, t)}{\partial y}
$$

where $p(x, y, t)$ is the original input; $g(x, y, t)$ is the intermediate filtered result obtained after equation (34) is used in the $\mathrm{x}$-direction; and $q(x, y, t)$ is the 3 -D final filtered result.

Because both equations (34) and (35) are 2-D variable-coefficient differential equations, we can solve them using the algorithm described in the 2-D case. However, in practice, we wish to avoid the costly transposition of 3-D seismic data when implementing the 3-D DEBF method so that the total computational efficiency can be further raised. Fortunately, we can alternately and recursively solve equation (34) and (35) between two adjacent traces in the $x$-direction and between two adjacent lines in the $y$-direction. The scheme is shown in Figure 8 . That is, we first get the intermediate result, $g(x+1, y+1, t)$, using $g(x, y+1, t), p(x, y+1, t)$ and $p(x+1, y+1, t)$, then get the final result, $q(x+1, y+1, t)$, using $q(x+1, y, t), g(x+1, y, t)$ and $g(x+1, y+1, t)$. As a result, we need not transpose 3-D seismic data from the inline direction to the crossline direction and then from the crossline direction to the inline direction for subsequent processings. To our knowledge, no other existing filtering methods can do 3-D filtering in this way. 
As before, implementation of 3-D low-dip-pass DEBF requires only subtraction of the high-dip-pass filtered result from the input data.

\subsection{Application to synthetic data}

Figure 9 is a sketch map of a 3-D common-shot experiment with 20 lines and 121 receivers on each line. Figure 10a simulates the direct arrival, along with four horizontal reflection events. Moveout of the direct arrival varies with both tirne and azimuth. Figure 10b, the filtered result of the 3-D DEBF method with $f_{N}=35 \mathrm{~Hz}$ and $V_{N}=6000 \mathrm{~m} / \mathrm{s}$, shows that the dipping event has been strongly suppressed in both the inline and the crossline directions, while the horizontal events have been preserved.

\section{CONCLUSIONS}

We have developed the method and shown examples of DEBF filtering in 1-D, 2-D, and 3-D. Inlike conventional filtering techniques, which use convolution operations or mathematical transforms, the method presented here directly solves variablecoefficient differential equations, and, as a result, the filtering parameters can vary at every time and space point. When we use a finite-difference algorithm to solve these filtering differential equations, the filtering processes are transformed into the solution of tridiaginal systems of equations, which have known, efficient solutions. From the principles and algorithms of these filtering methods and the examples with synthetic and field seismic data we have done, we conclude that the DEBF methods have the following characteristics.

1. DEBF filter parameters can arbitrarily vary with both time and space.

2. Because their operators are short, the cc mputational efficiency of DEBF is quite high. (Its speed for 2-D processing is comparable to that of $f-k$ filtering, which lacks flexibility for addressing non-stationarity.) Furthermore, because we use an implicit solution in the time direction and a recursive approach in the space direction, their impulse responses, in effect, are long.

3. Because these DEBF methods process seismic data trace-by-trace, they need not do any transposition of seismic data. This further raises the total computational efficiency, especially for 3-D filtering. Moreover, these methods therefore require less inner computer memory.

4. DEBF methods do not have any specific requirements for the length and the number of input traces.

5. Because these methods need relatively little core memory and are relatively efficient, they are well suited for use not only on large computers, but also on microcomputers and workstations. 
6. Although not shown here, they can be adapted to treat seismic data with nonuniform space sampling intervals. While non-uniform sampling makes the computation more complicated, it poses no fundamental limitation.

7. 2-D and $3-D$ dip filters cannot distinguish between events with positive dips and those with negative dips.

8. For the simple filter examples considered here, the cut-off boundary of DEBF is not as steep as that readily obtainable with $f-k$ filtering.

\section{ACKNOWLEDGEMENTS}

The authors gratefully acknowledge the support of the sponsors of the Consortium Project on Seismic Inverse Methods for Complex Structures at the Center for Wave Phenomena, Colorado School of Mines. This project was also partially supported by the United States Department of Energy, grant number DE-FG02-89ER14079. (This support does not constitute an endorsement by DOE of the views expressed in this paper.)

\section{REFERENCES}

Anderson, R. G. and McMechan, G. A., 1988, Noise-adaptive filtering of seismic shot records: geophysics, 53, 638-649.

Claerbout, J. F., 1985, Imaging the earth's interior: Blackwell Scientific Publications.

Hardage, B. A., 1983, Vertical seismic profiling: Geophysical Press, London-Amsterdam.

Katz, A. and Katz, S., 1990, Multichannel adaptive filters: 60th Annual International Meeting, Society of Exploration Geophysicists, Expanded Abstracts, 1697-1700.

McMechan G. A. and Sun R., 1991, Depth filtering of first breaks and ground roll: Geophysics, 56, 390-396.

Noponen, I. and Keeney, J., 1983, Attenuation of waterborne coherent noise by application of hyperbolic velocity filtering during the tau-p transform: presented at 53rd Annual International Meeting, Socicty of Exploration Geophysicists.

Treitel, S., Shanks, J., and Frasier, C., 1967, Some aspects of fan filtering: Geophysics, 32, 789-800.

Wiggins, R., 1966, $\omega-k$ filter design: Geophysical Prospecting, 14, 427-440. 


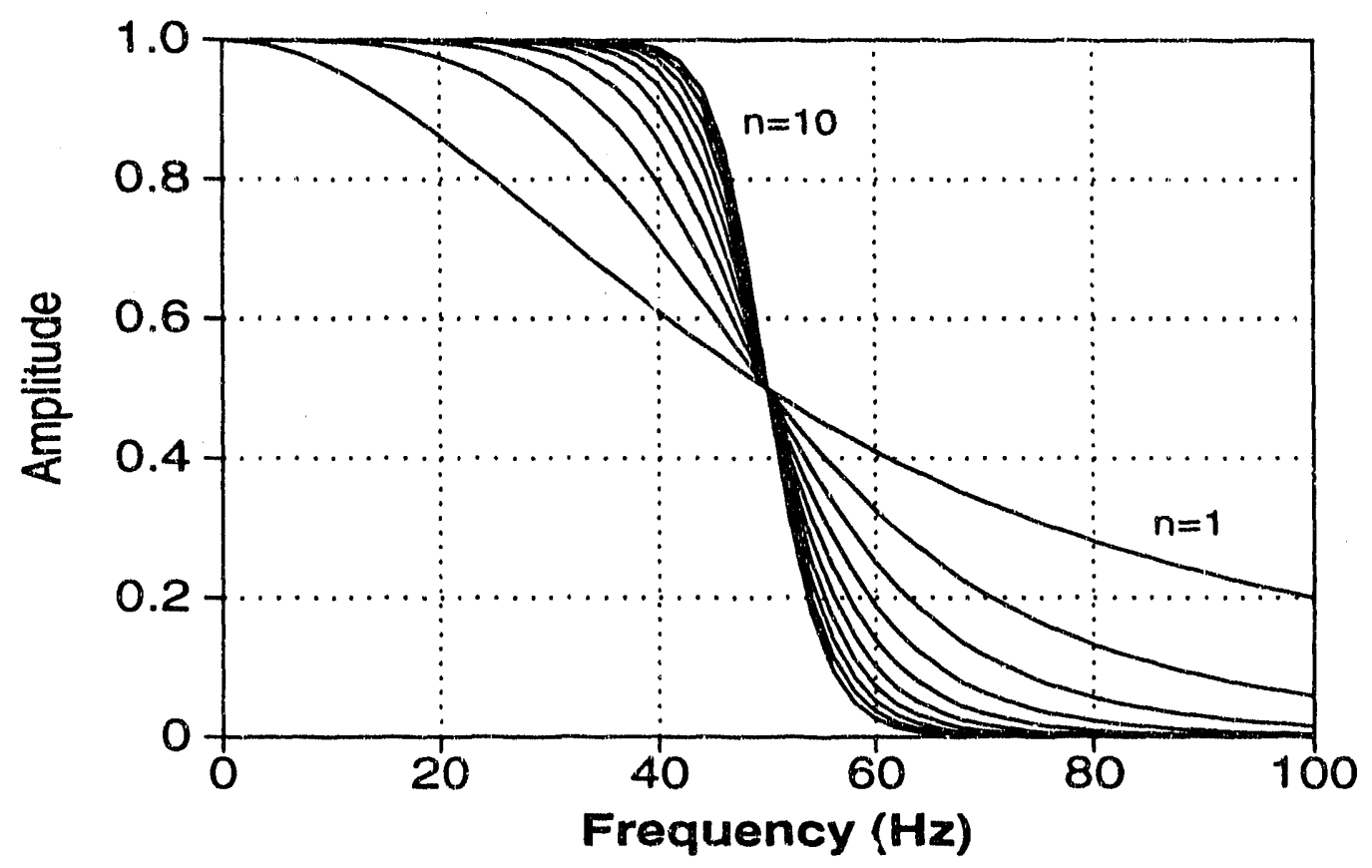

Fig. 1. Amplitude curves of the low-frequency-pass filter $H_{1}(\omega)$ for $n=1,2,3, \cdots, 10$.

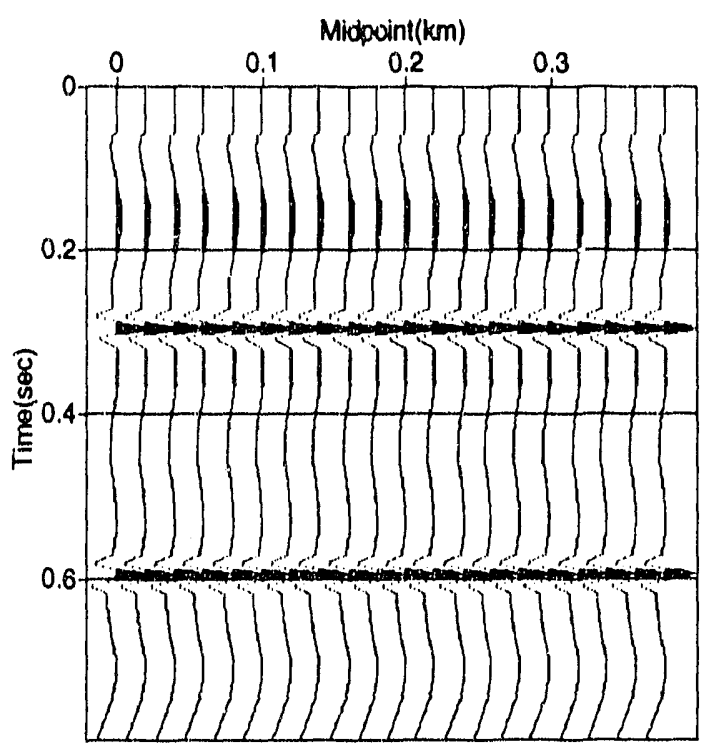

(a)

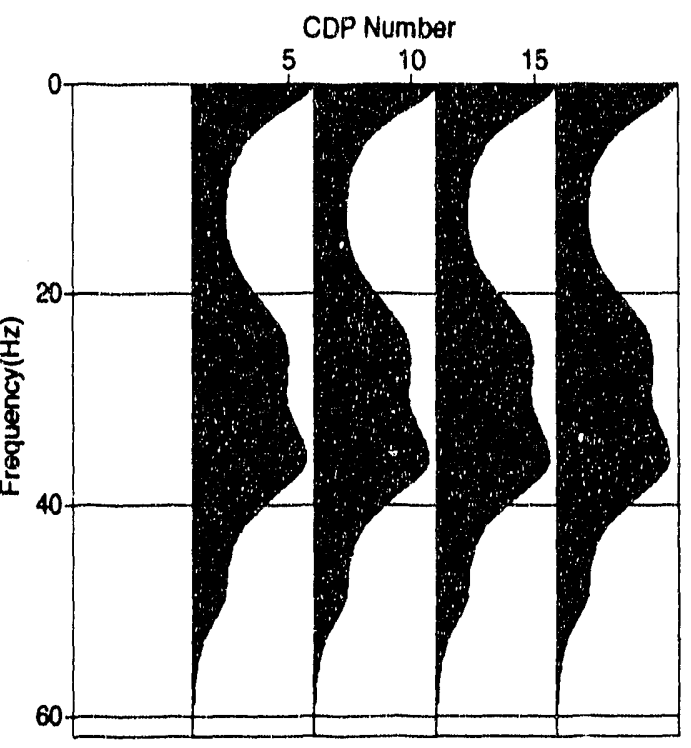

(b)

FIG. 2. (a) Synthetic data contaminated by 1-10 Hz low-frequency "noise". (b) Amplitude spectra of four of the traces in (a). 


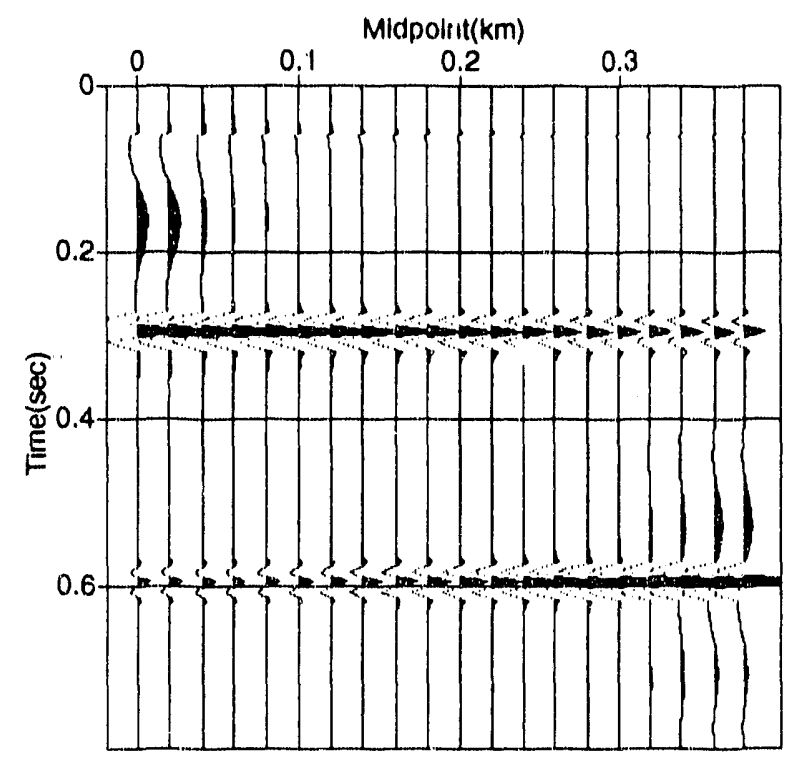

(a)

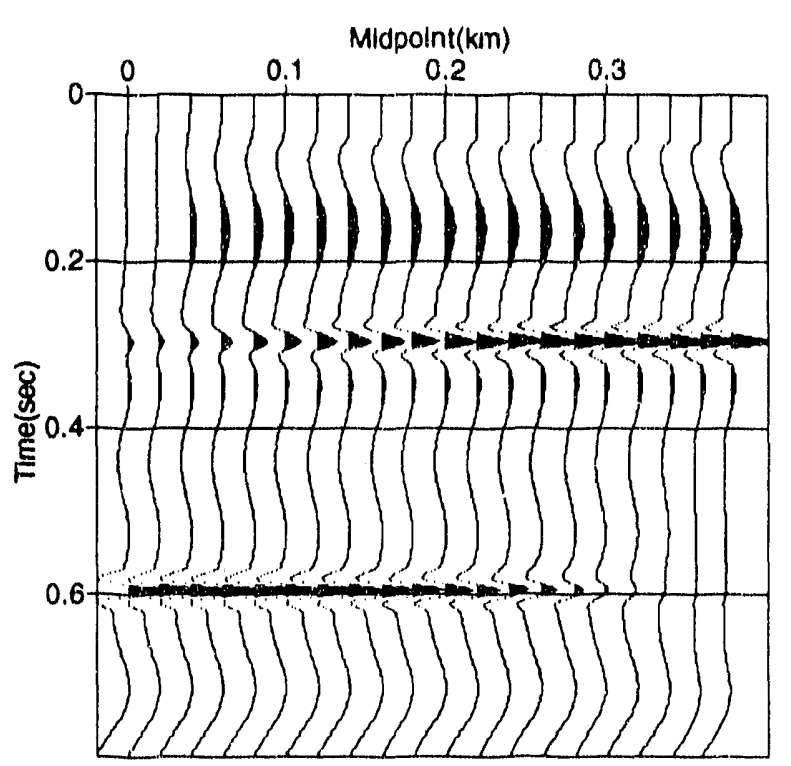

(b)

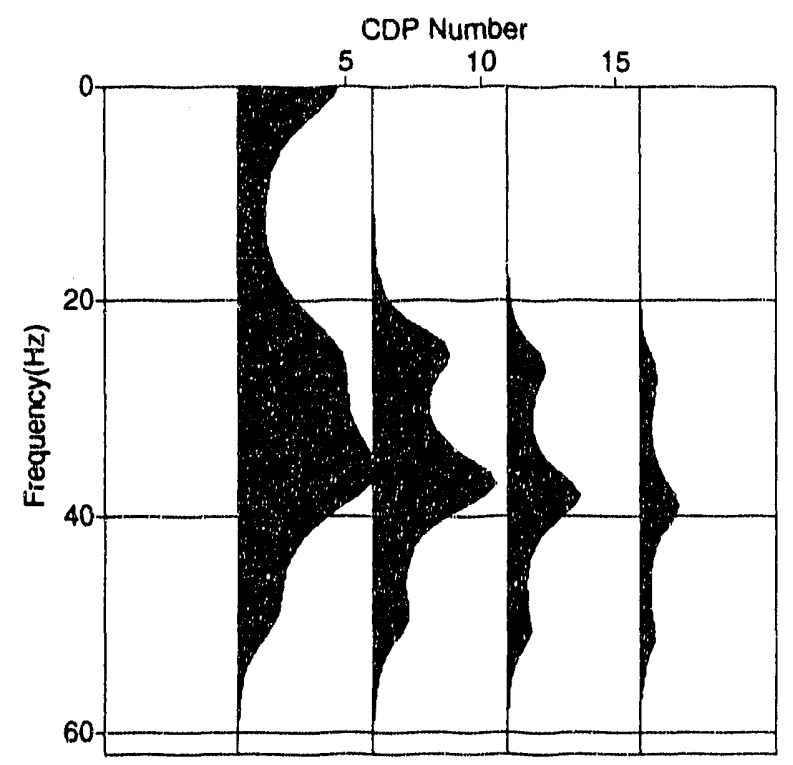

(c)

FIG. 3. (a) Time- and space-variable filtered result of Figure 2a. The low cut-off frequencies are both time- and space-variable. (b) Time- and space-variable filtered "noise" section. (c) Arnplitude spectra of the time- and space-variable filtered section (a) within a $400 \mathrm{~ms}$ time window centered on $200 \mathrm{~ms}$. 


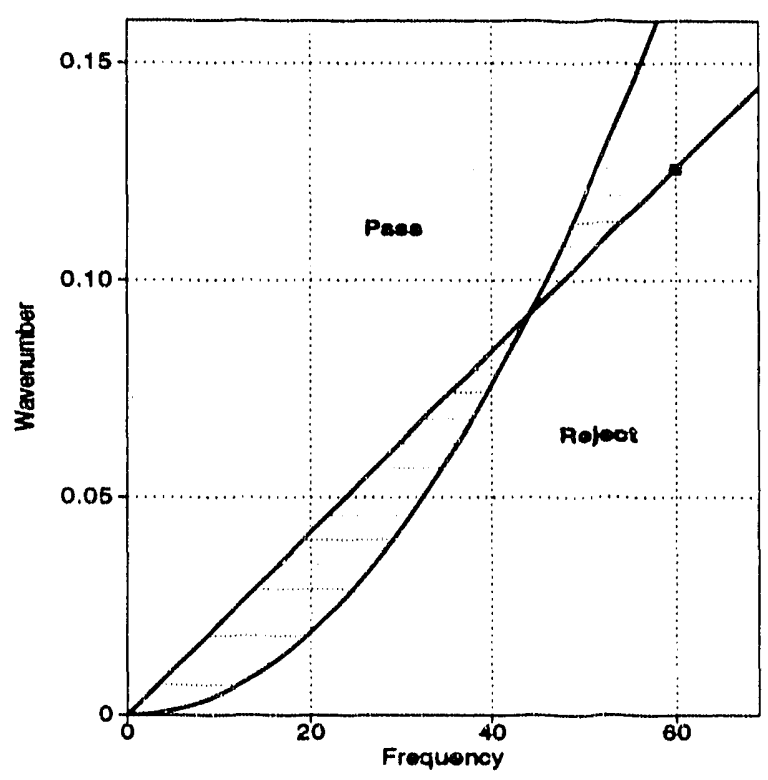

(a)

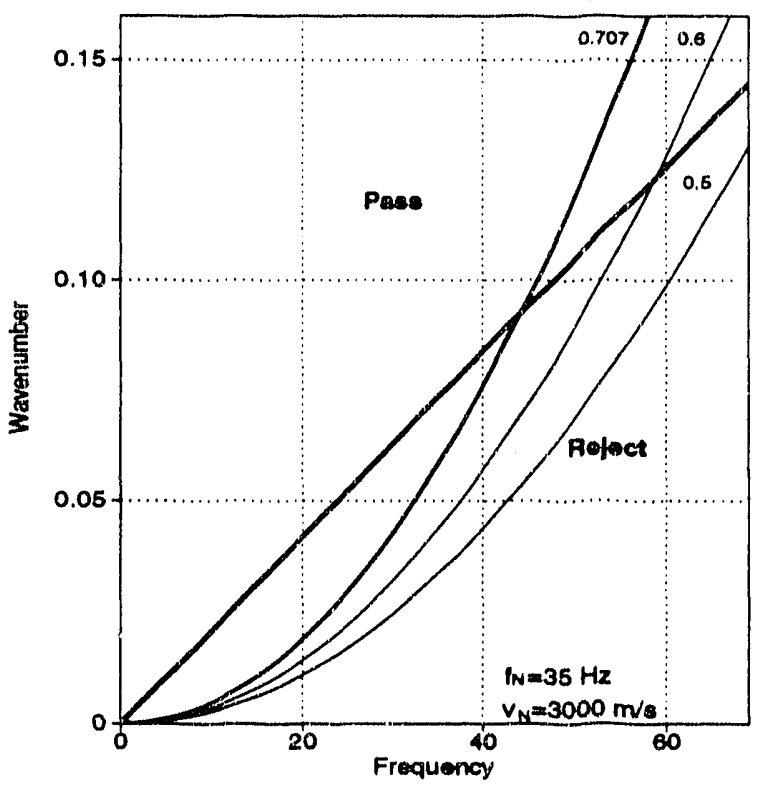

(b)

FIG. 4. (a) Ar a between the straight-line boundary of an ideal dip filter and the parabolic boundary of the 2-D high-dip-pass DEBF filter. (b) Straight-line boundary of an ideal dip filter and three contours of the amplitude spectrum $\left|H_{1}\left(k_{x}, y, \omega\right)\right|$. 


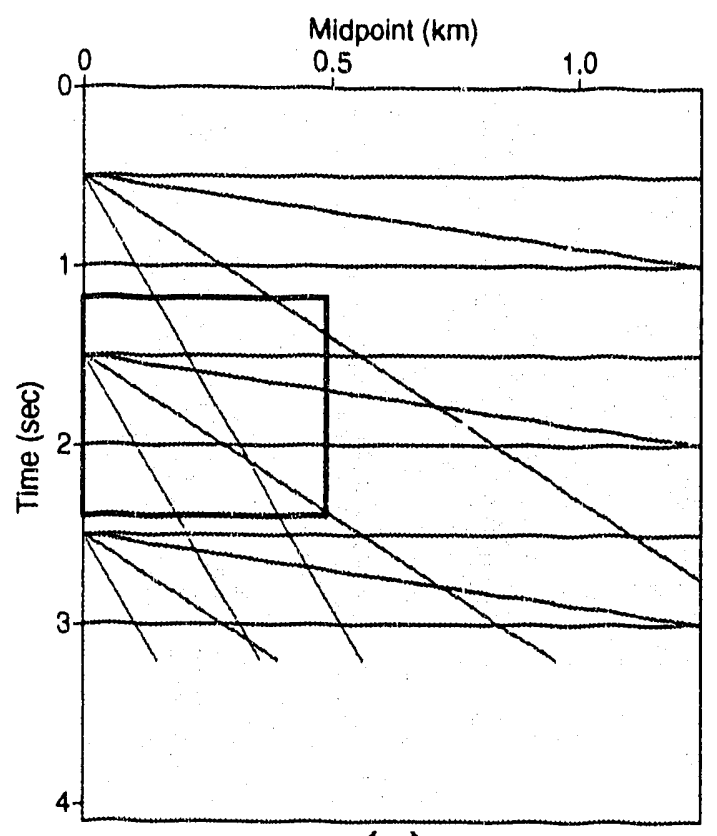

(a)

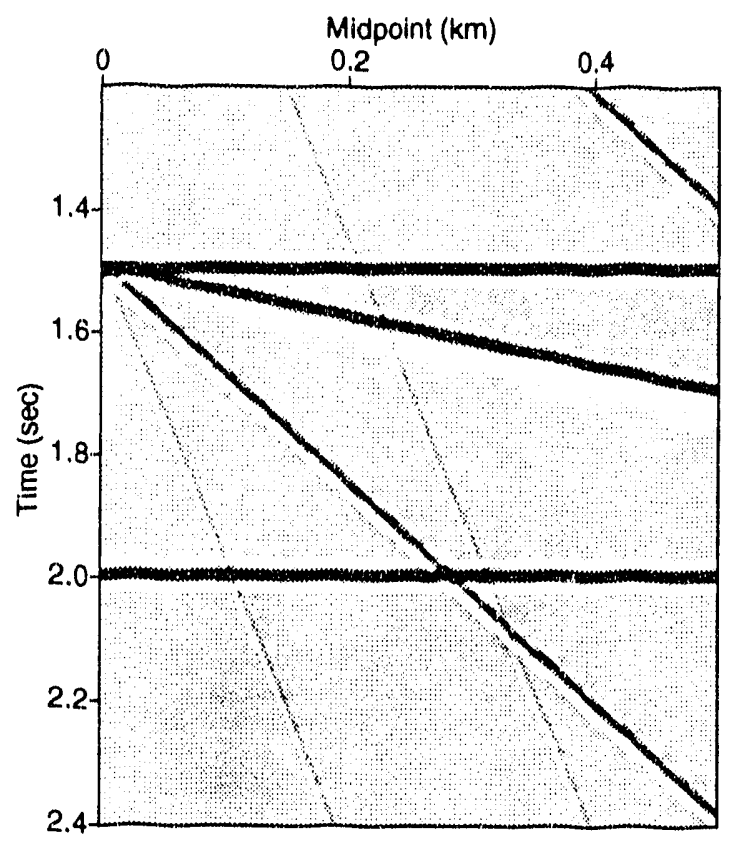

(c)

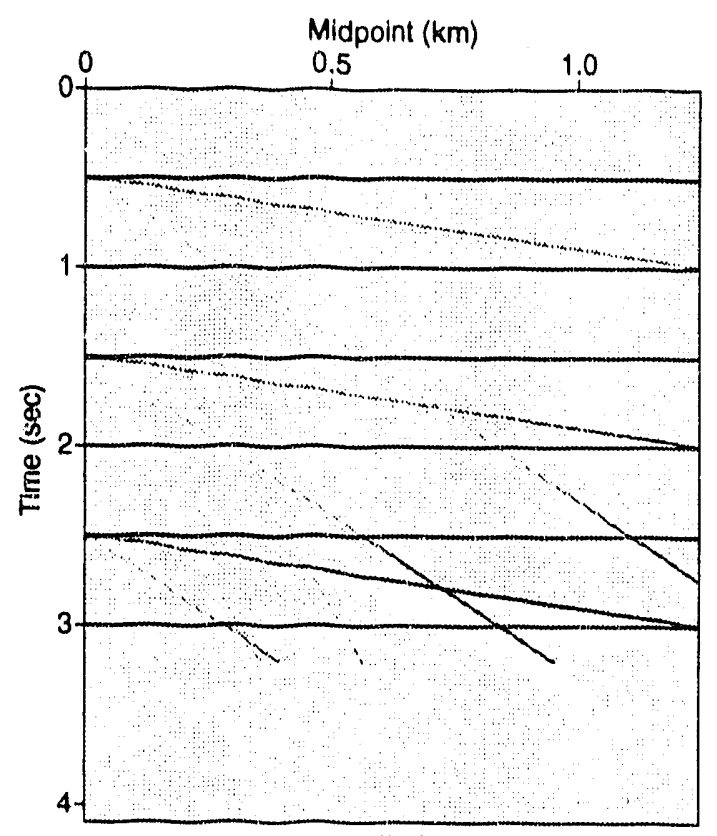

(b)

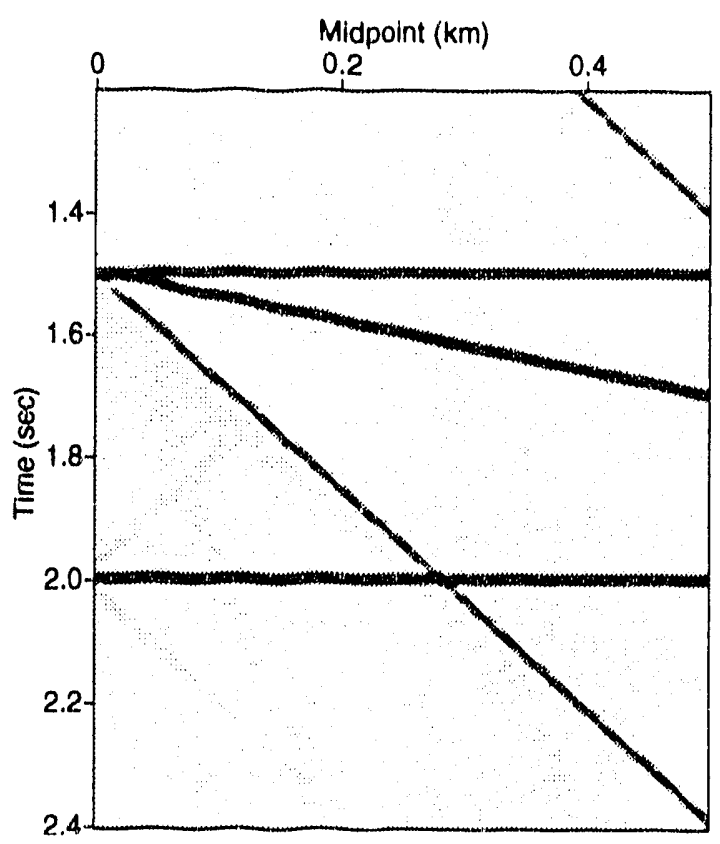

(d)

FIG. 5. (a) Synthetic seismic section with six horizontal and nine dipping events. (b) Time- and space-variable DEB low-dip-pass filtered section of (a). (c) Detail of DEB low-dip-pass filtered section of (a) to compare with (d). (d) Detail of low-dippass filtered section of (a) obtained by using $f-k$ filtering. 


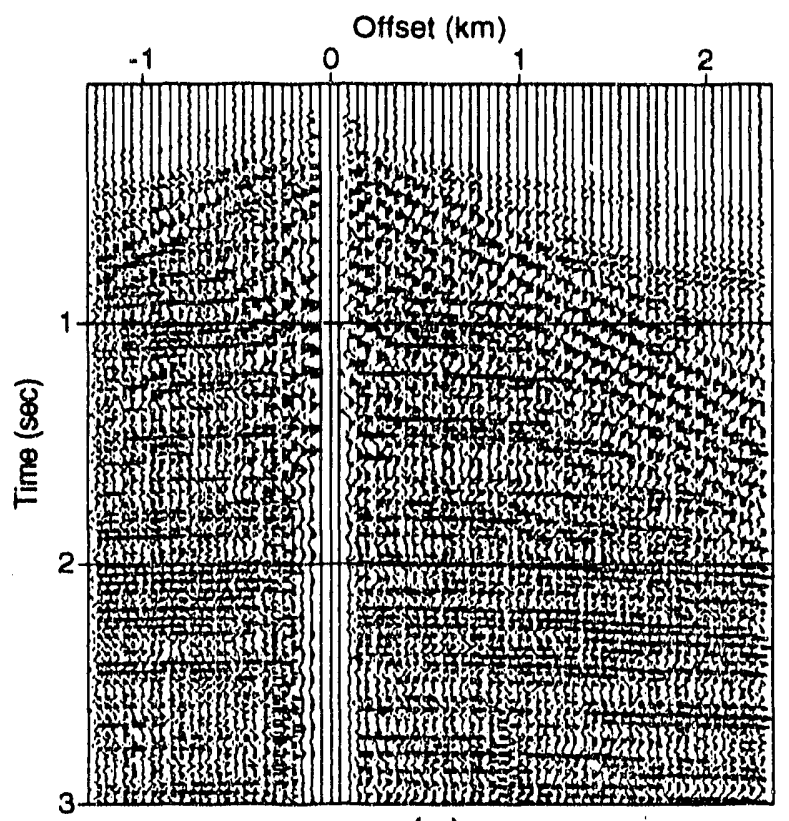

(a)

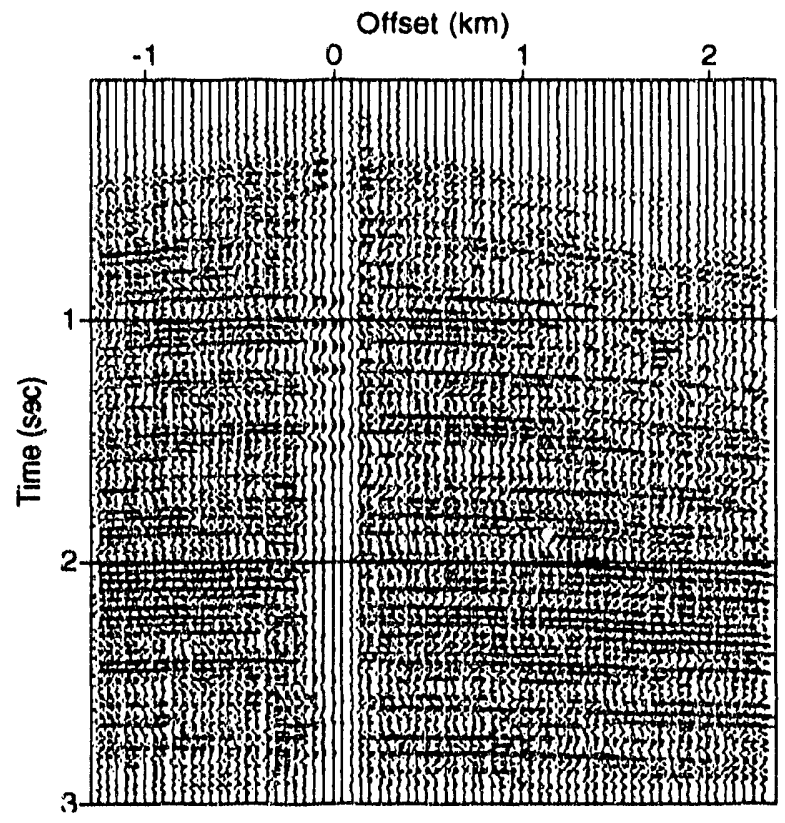

(b)

FIG. 6. (a) Field shot record with strong, dipping coherent noises in the upper part. (b) Time- and space-variable filtered result of (a). All dipping noises have been removed, improving the signal-to-noise ratio. 


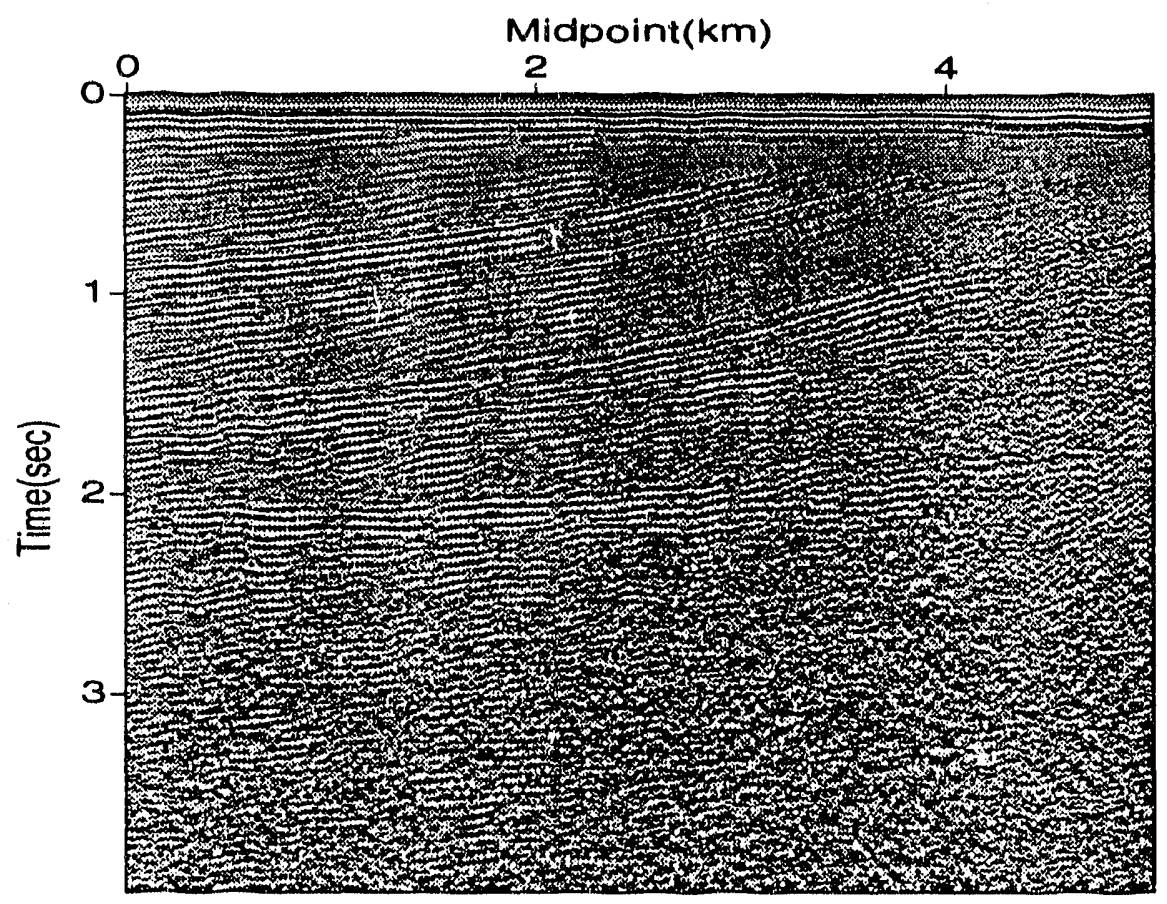

(a)

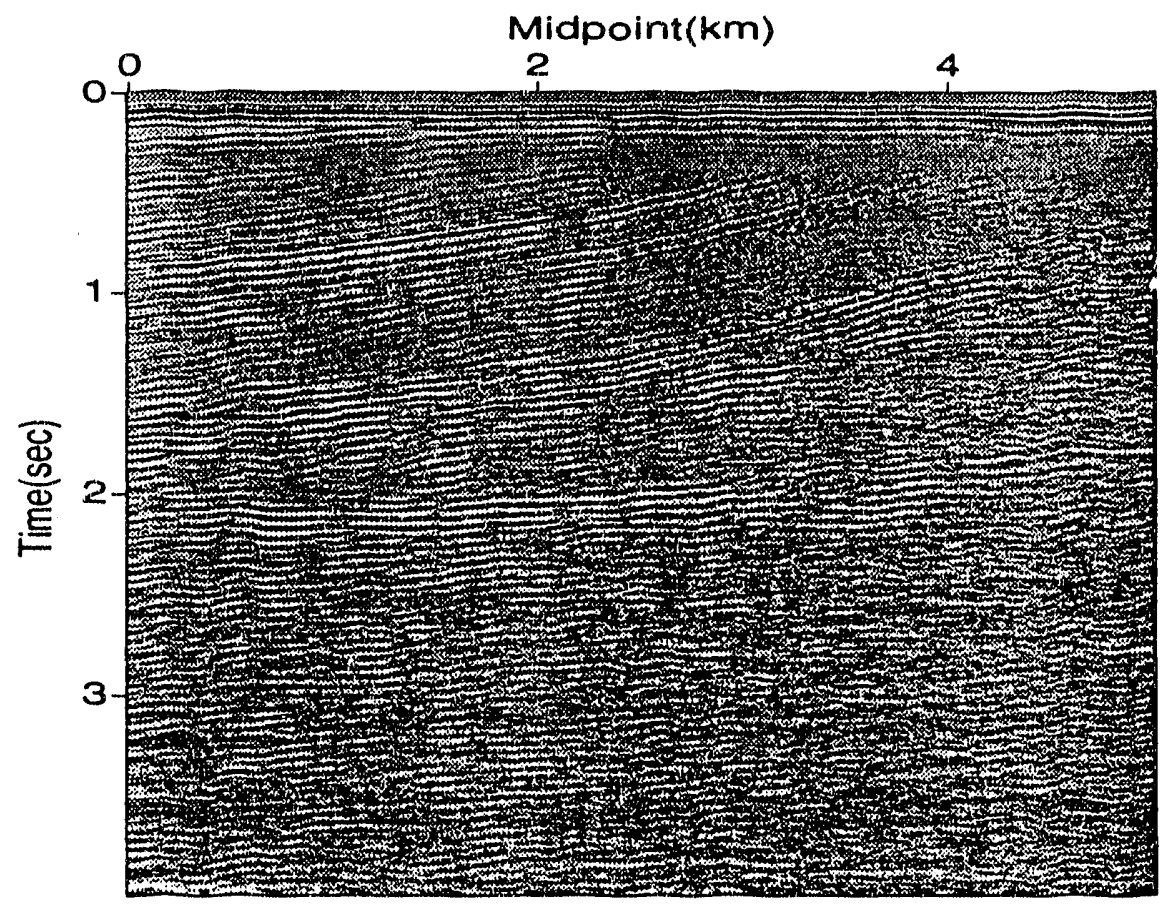

(b)

Fig. 7. (a) Common-offset field seismic section with some dipping background noise. (b) DEB filtered section of (a). 


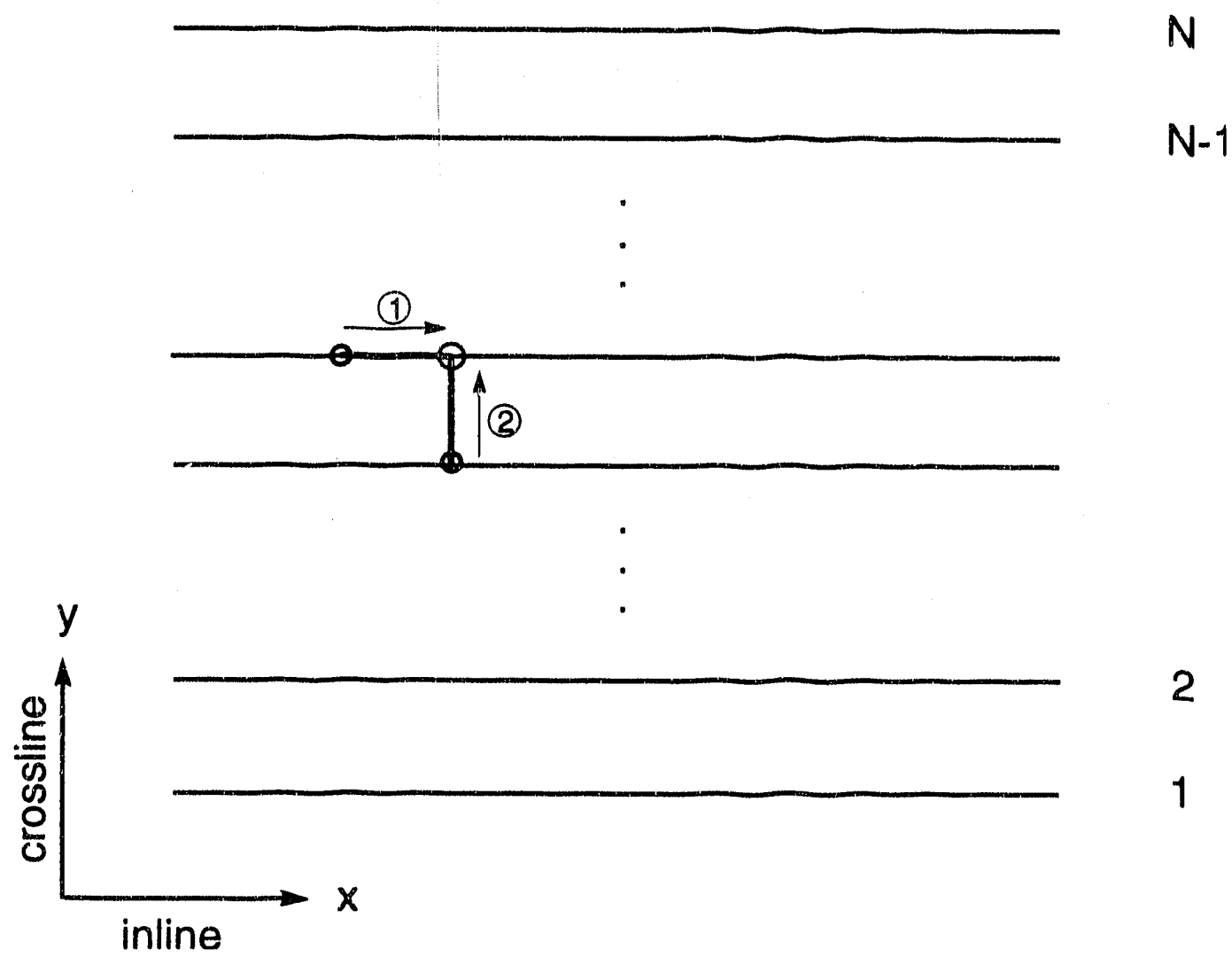

FIG. 8. Sketch plan view showing lines of data used in an implementation of the 3-D DEBF method. 


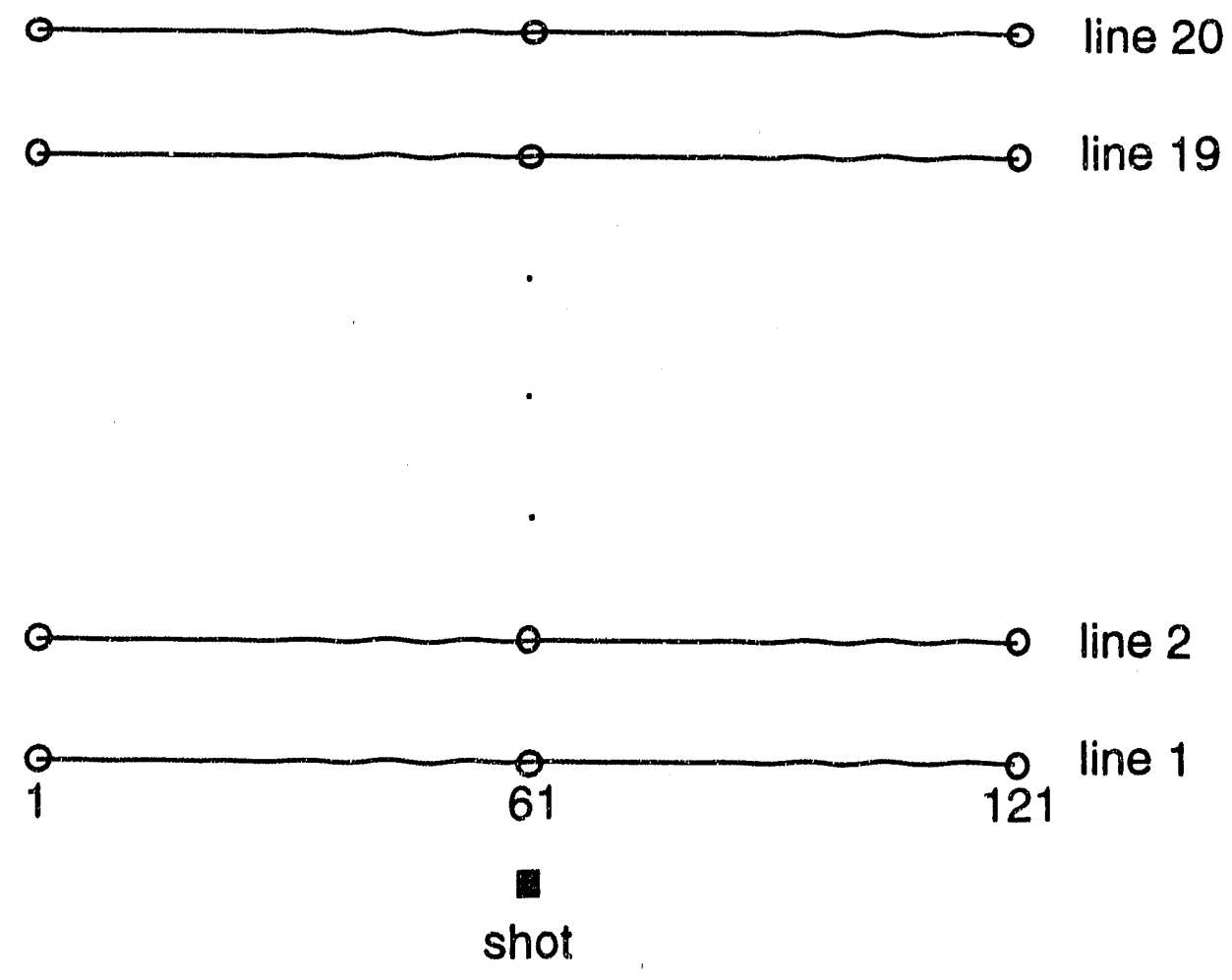

FIG. 9. Plan-view sketch map of a 3-D common-shot experiment. 


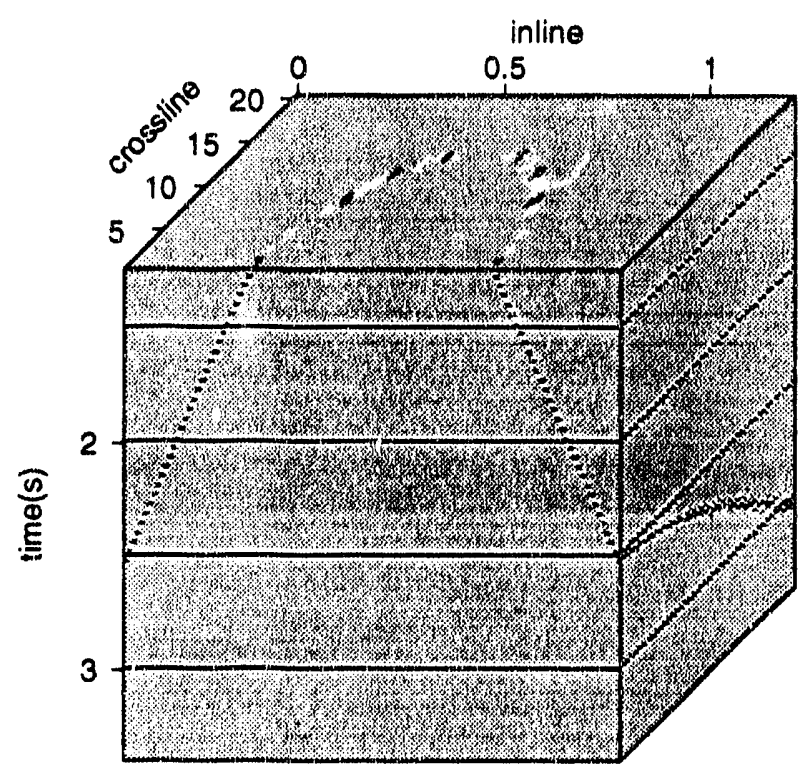

(a)

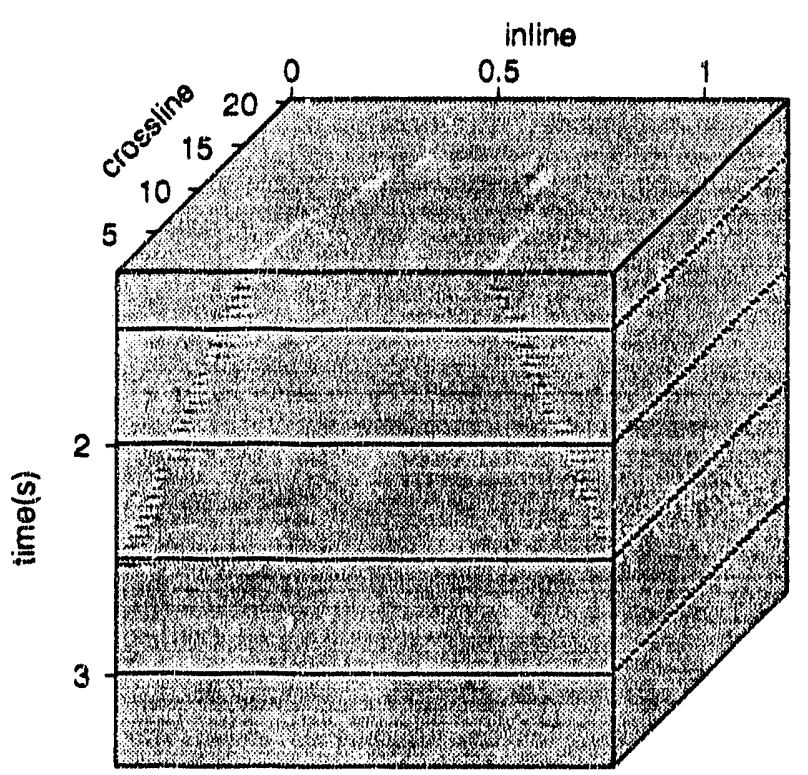

(b)

FIG. 10. (a) Part of a synthetic seismic data set. The dipping event simulates the direct wave in a 3-D case. (b) 3-D low-dip-pass filtered result of (a). The dipping event has been strongly suppressed. 


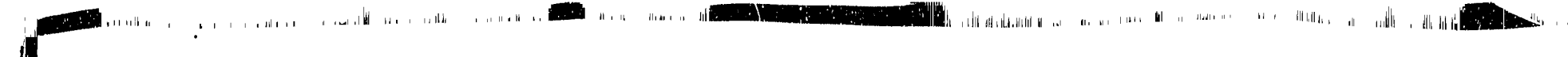
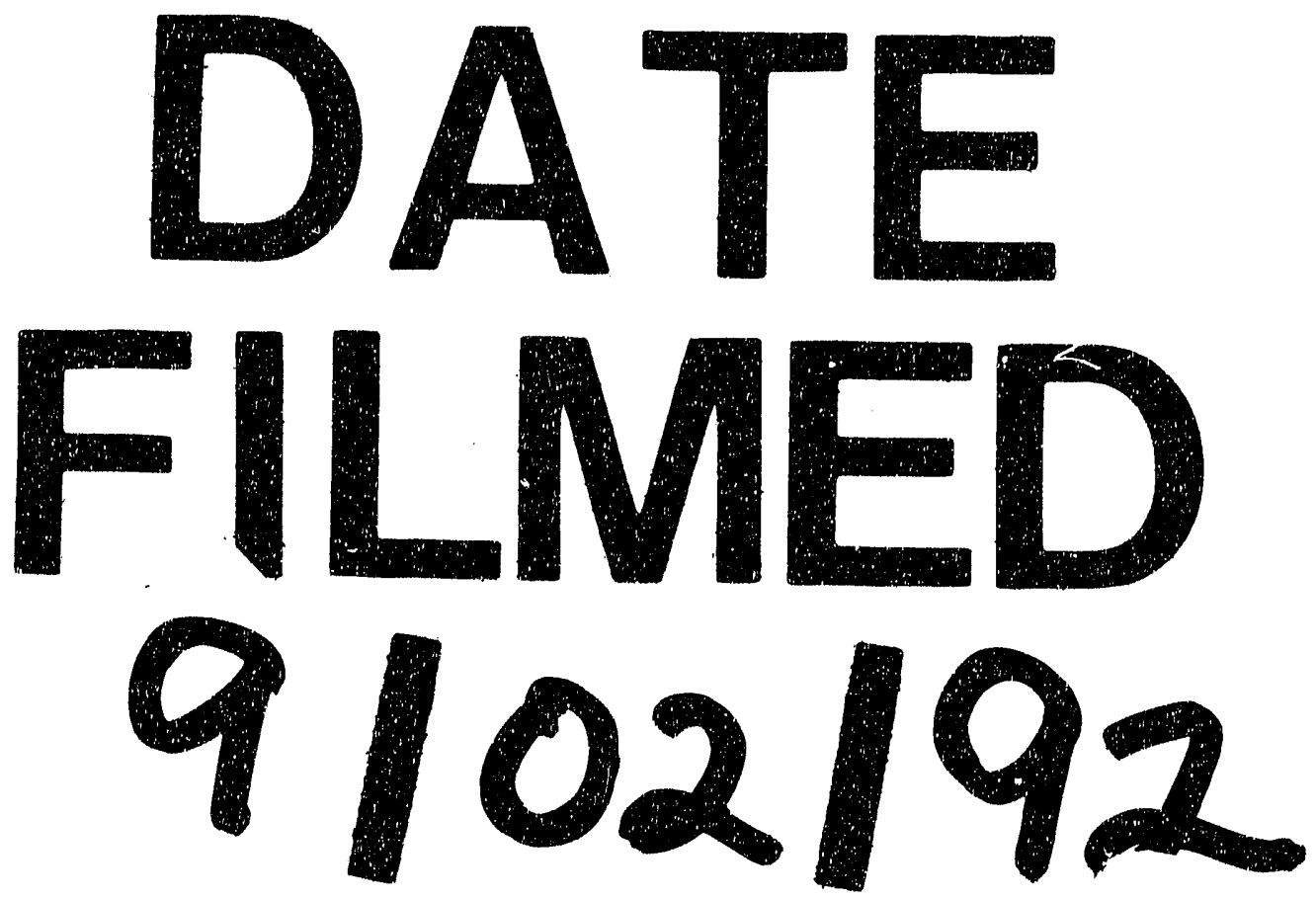


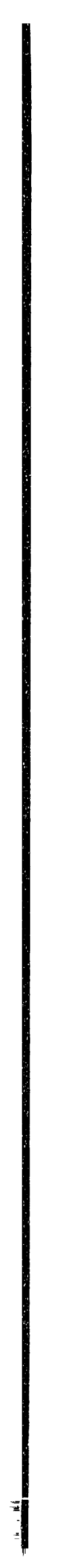

